\title{
Photometry of Long Period Variables in the Magellanic Clouds
}

\section{J. BUTLER (Dublin)}

A photographic survey of variable stars in the Magellanic Clouds is currently in progress at Dunsink Observatory, using photographic material obtained with the ADH telescope. In the course of this investigation a number of Long Period Variables have been measured, most of which lie in the LMC. Several are previously known Harvard Variables (prefixed HV) but some are new discoveries (prefixed DV). The co-ordinates of the new Long Period Variables are listed in Table $\mathrm{I}$.

Table I

\begin{tabular}{|c|c|c|c|c|}
\hline Name & $\mathrm{RA}(1875)$ & $\operatorname{DEC}(1875)$ & $\mathrm{x}$ & y \\
\hline DV 140 & $4 h_{57 m} 23$ s & $-68^{\circ} 09^{\prime} 05^{\prime \prime}$ & 2537 & 13233 \\
\hline DV 151 & $\begin{array}{lll}459 & 41.1\end{array}$ & $-68 \quad 0432$ & 3282 & 13584 \\
\hline DV 205 & $\begin{array}{ll}508 \quad 40.0 \\
\end{array}$ & $-66 \quad 5821$ & 6076 & 17812 \\
\hline DV 225 & $\begin{array}{lll}511 & 07.6\end{array}$ & $-67 \quad 2730$ & 7035 & 16099 \\
\hline DV 240 & $\begin{array}{lll}5 & 12 & 44.8\end{array}$ & $\begin{array}{lll}-68 & 1415\end{array}$ & 7719 & 13303 \\
\hline
\end{tabular}

Magnitudes were derived from iris diaphragm photometry of about thirty plates in $V$, and $B$, each of which was calibrated using a large number of photoelectric standards, BUTLER (1972a). The reduction of the iris readings to magnitude was made using a computerized technique which makes allowance for the colour of the star, background fog, and various instrumental effects, BUTLER (1972b).

All the Long Period Variables observed fall into two distinct groups, one with periods less than two hundred days and the other with periods greater than three hundred days. As the total period of the observations was only $\sim 500$ days the variables in the longer period group did not have sufficient coverage to allow a reasonable estimate of the period to be made. Thus for the variables in the short period group the observations are plotted in Fig. 1 against phase, and for variables in the long period group the observations are plotted in Fig. 2 against Julian Date.

For the SMC Long Period Variables HV 12122 and HV 2112 the periods given by PAYNEGAPOSCHKIN and GAPOSCHKIN (1966) have been adopted. The period given by the GAPOSCHKINs for HV 11366 does not fit the observations made of this star, but as the amplitude is small this may be due, at least partially, to scatter in the observations.

Periods were not available in the literature for the LMC Long Period Variables. Consequently their periods were estimated using a period finding computer program similar to that described by LAFLER and KINMAN (1965), but again because the observations only span two observing seasons, the derived periods cannot be considered in any way accurate.

In Table 2 the Long Period Variables which have light curves are listed with the epoch of maximum, the approximate period, the magnitudes and colour at maximum, and the observed amplitudes in $\mathrm{V}$ and $\mathrm{B}$. Uncertain quantities are bracketted. For some variables - notably those in the short-period group - the minima of the light curves are below the plate limit and only the maxima can be determined with any degree of certainty. Thus for these variables the amplitudes given in Table 2 are minimum values only. 

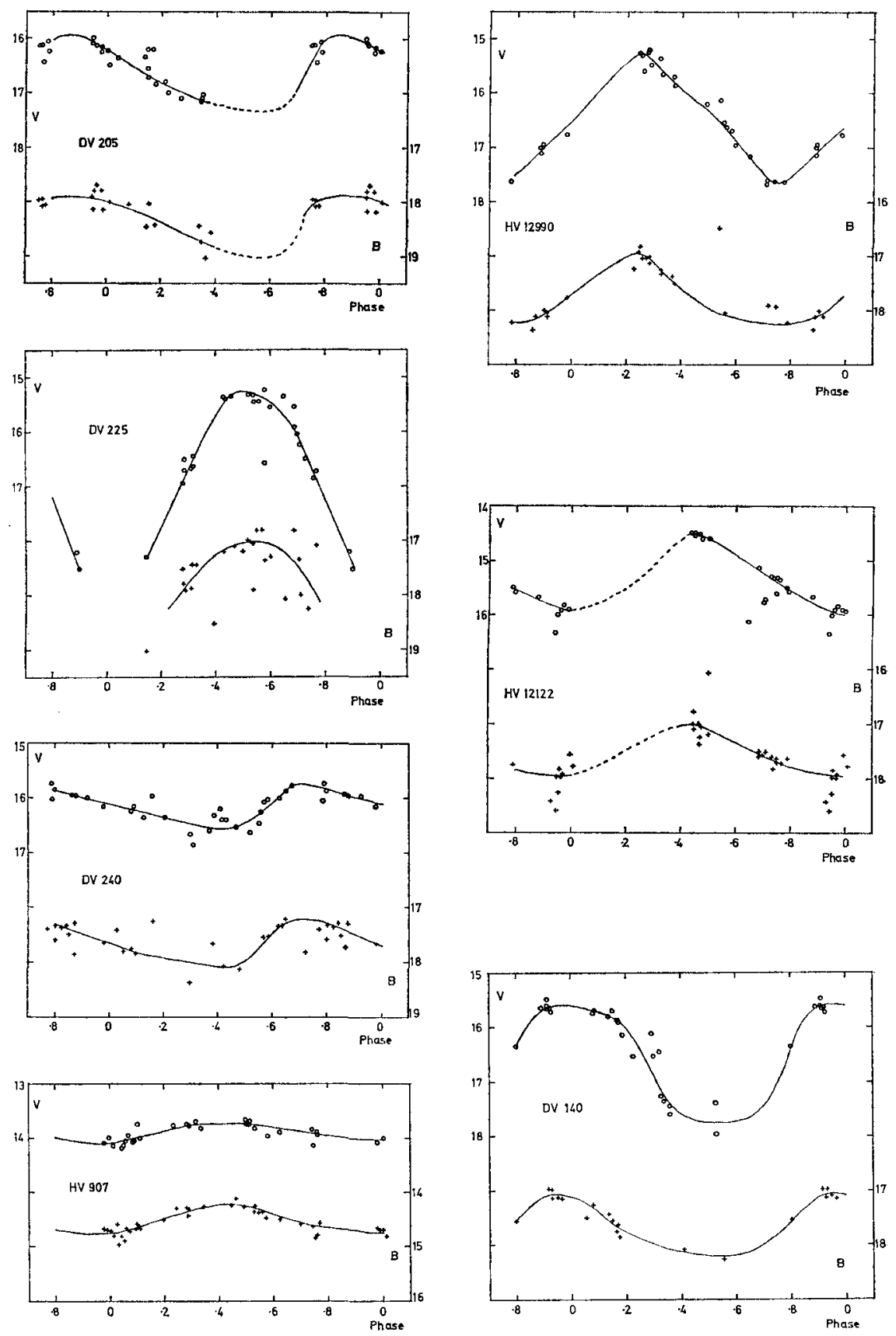

Fig. 1: Observations Plotted against Phase for Variables with Periods $<200$ days. Circles V, Crosses B. 

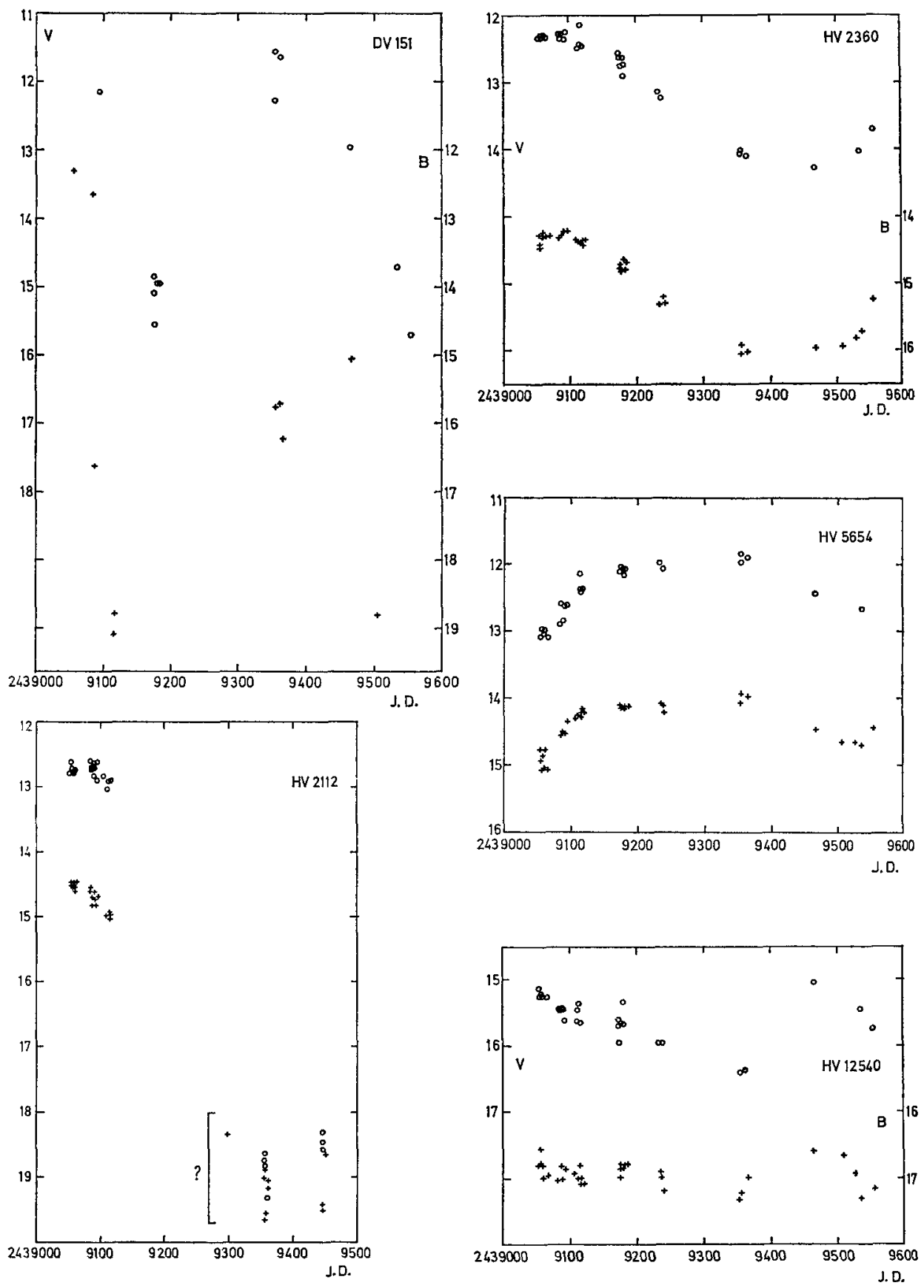

Fig. 2: Observations Plotted against Julian Date for Variables with Periods $>300$ days. Circles V, Crosses B. 
Table 2

\begin{tabular}{|c|c|c|c|c|c|c|}
\hline & & meters for & Long Period V & ariables & & \\
\hline Name & $\begin{array}{l}\text { Epoch of } \\
\text { maximum }\end{array}$ & Period & $V_{\max } B_{\max }$ & $\mathrm{B}_{\max }-\mathrm{V}_{\max }$ & $A_{v}$ & $A_{b}$ \\
\hline SMC & JD & days & & magnitud & es & \\
\hline HV 2112 & - & 608 & $(12.7)(14.5)$ & (1.8) & 6.0 & 5.0 \\
\hline HV 12122 & - & 120 & $(14.5)(17.0)$ & (2.5) & 1.8 & 1.5 \\
\hline LMC & & & & & & \\
\hline HV 5654 & 2439360 & $>500$ & 11.8913 .98 & 2.09 & 1.2 & 1.0 \\
\hline HV 2360 & 2439080 & $(520)$ & $12.30 \quad 14.30$ & 2.00 & 2.1 & 1.8 \\
\hline HV 12540 & $(2439450)$ & (450) & $(15.2)(16.6)$ & (1.4) & 1.2 & 0.6 \\
\hline DV $\quad 151$ & & (350) & & & 6.0 & 6.0 \\
\hline HV 12990 & 2439174 & 169 & $\begin{array}{lll}15.25 & 16.86\end{array}$ & 1.61 & 2.4 & 1.2 \\
\hline 225 & 2439523 & 157 & 15.2817 .05 & 1.77 & 3.0 & 2.5 \\
\hline 140 & 2439178 & 156 & $\begin{array}{llll}15.60 & 17.07\end{array}$ & 1.47 & 2.4 & 1.2 \\
\hline 205 & 2439196 & 151 & $15.97 \quad 17.90$ & 1.93 & 1.2 & 1.2 \\
\hline 907 & 2439103 & 111.8 & $13.72 \quad 14.25$ & 0.53 & 0.45 & 0.6 \\
\hline 240 & 2439107 & $(78.7)$ & $15.78 \quad 17.28$ & 1.50 & 0.9 & 0.9 \\
\hline
\end{tabular}

Three of the variables listed in Table 2 have been observed photometrically by other observers namely, HV 2112, and HV 12122 in the SMC by the GAPOSCHKINs, and HV 12990 in the LMC by HODGE and WRIGHT (1969). The GAPOSCHKINS gave values of $\mathrm{m}_{0}$ (photographic magnitude corrected for absorption) of 13.0 for HV 2112 and 14.52 for HV 12122. Both these values are considerably brighter than the corresponding values of $B_{\max }$ in Table 2. It is probable that the maxima have been missed for these stars, but, as the GAPOSCHKINs have not published the light curves or the amount of the correction for absorption that they have applied, no detailed comparison can be made. For HV 12990, HODGE and WRIGHT found a period of 161.6 days, and gave $V_{\max }=14.78$, and $B_{\max }=16.68$. The agreement in $B$ is reasonable but there is again an appreciable discrepancy in V. The results of both the GAPOSCHKINs and of HODGE and WRIGHT are based on observations over a much longer period than are those presented here.

Two of the variables DV 151 in the LMC and HV 2112 in the SMC belong to the class of very large amplitude variables observed spectroscopically by LLOYD EVANS (1971).

For all LMC variables with reasonably well defined maxima the magnitudes at maximum in $\mathrm{V}$ are plotted against period in Fig. 3.

The two LMC variables HV 2360 and HV 5654 are, to judge from their period and magnitude at maximum, similar to the Long Period Variables found by the GAPOSCHKINS in the SMC. Assuming a true distance modulus of 18.66 for the $\mathrm{LMC}$ and an interstellar reddening of .05 mag in ( $\mathrm{B}-\mathrm{V})$, their mean absolute magnitude at maximum is 6.73 in $\mathrm{V}$, and -4.72 in $B$. These stars do not appear to have their counterparts amongst the Galactic Long Period Variables.

HV 907 has a nearly sinusoidal light curve of small amplitude. Its magnitude at maximum is brighter than the rest of the short-period group in the LMC by more than two magnitudes, and it is possibly a foreground star.

The other five members of the short period group have comparable magnitudes at maximum. Assuming the distance modulus and absorption as above, the mean absolute magnitudes at maximum of the four LMC stars with $P \sim 160$ days are as follows:

$$
\begin{aligned}
& M_{V}(\max )=-3.30 \pm .17(\mathrm{rms}) \\
& M_{B}(\max )=-1.64 \pm .23
\end{aligned}
$$

Including the lower amplitude and shorter period variable DV 240 we obtain:

$$
\begin{aligned}
& M_{\mathrm{V}}(\max )=-3.25 \pm .14 \\
& M_{\mathrm{B}}(\max )=-1.63 \pm .18
\end{aligned}
$$




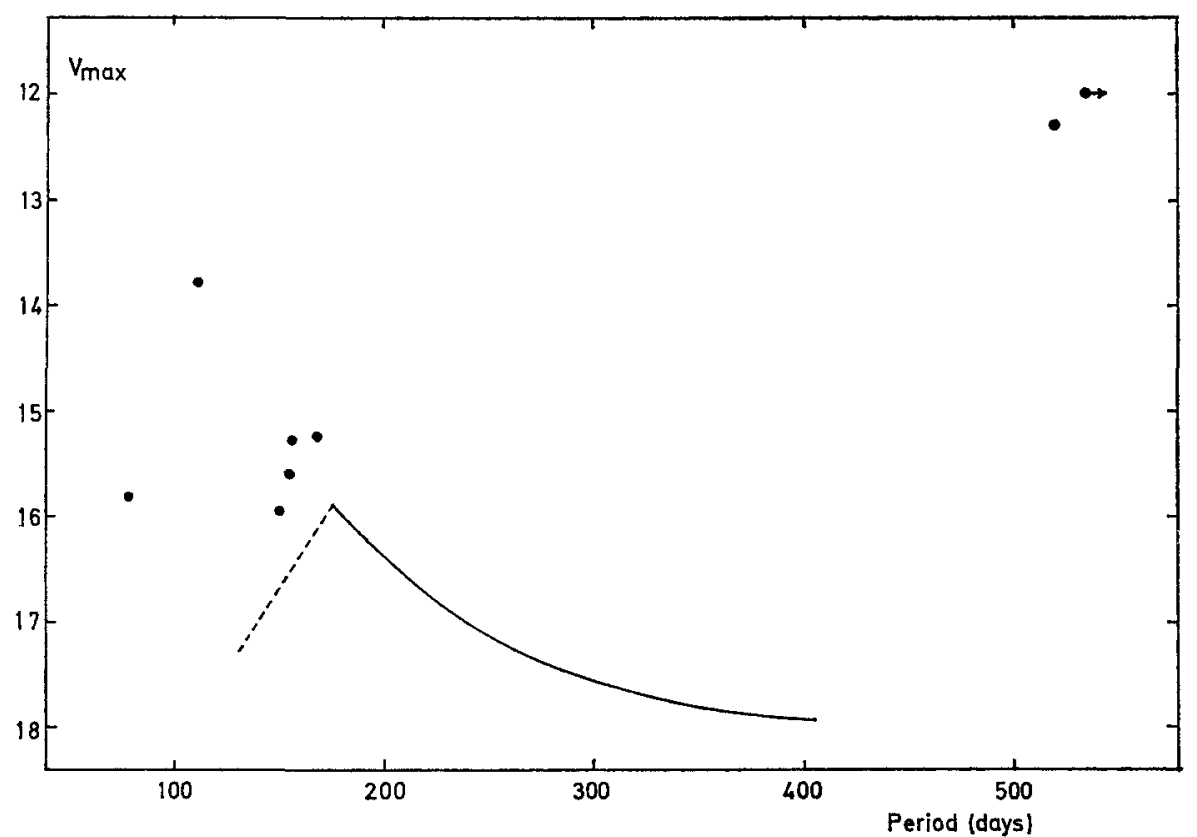

Fig. 3: $V_{\max }$ against Period for Long Period Variables in the LMC.

In order to compare the Long Period Variables in the LMC with the Mira Variables in the Galaxy, the Period - Mv (max) Relation determined from statistical parallaxes by CLAYTON and FEAST (1969), is shown in Fig. 3 shifted by an apparent distance modulus of $18.82 \mathrm{mag}$. The LMC variables with periods less than 200 days lie approximately 0.8 magnitudes above the Galactic relation as given by these authors. The discrepancy is caused by the sudden drop in $M_{V}$ for the shortest period group of variables analysed by CLAYTON and FEAST. The LMC stars appear to lie on a continuation of the long period section of CLAY. TON and FEAST's Period Luminosity Relation.

FEAST (1965) has shown that the SRd variables, which are present in the spherical component of the Galaxy and in some globular clusters, form a group with similar periods to the short period Miras but with absolute magnitudes more than one magnitude brighter. FEAST has postulated that the SRd variables are fundamental pulsators whereas the normal Mira variables are first harmonic pulsators at this period.

STOTHERS (1963) has obtained the absolute magnitude at maximum of Long Period Variables in Galactic globular clusters, using either RR Lyraes or their Colour Magnitude Diagrams to determine their distance moduli. Whilst it has not been possible to classify each variable unambiguously as $\mathrm{SRd}$, the data for the variables with $90<\mathrm{P}<170$ gives

$$
\mathrm{M}_{\mathrm{pg}}(\max ) \sim-2.0
$$

For the same stars FEAST (1965) has tentatively proposed a mean visual absolute magnitude

$$
\mathrm{MV}_{\mathrm{V}}(\max ) \sim-3.0
$$

The agreement with the values given above for the $L M C$ variables is reasonable considering (a) the statistical scatter present, (b) that the mean period of the Galactic group is less than that of the LMC group, (c) the possibility that both groups are to some extent inhomogeneous. 
Thus from their luminosities, periods and amplitudes, it seems that the group of fous LMC variables with periods around 160 days may be SRd, but their colours are much redder than would be expected for the $G, K$ type spectral class normally assigned to Galactic SRd stars (KUKARKIN et al., 1969). However, because of the influence of TiO absorption in the spectra of these stars, their colours may be a misleading guide to their spectral class. BAUM (1952) has found that late type giants in $\mathrm{M92}$ have a much earlier spectral class than would be inferred from their colours, and, as STOTHERS (1963) has pointed out, this colour excess could be a result of the lower Tio abundance that would be expected in globular clusters. If the LMC Long Period Variables with $\mathrm{P} \sim 160$ days were assumed to be SRd their apparent redness could also be explained by the metal deficiency that is expected for stars of this type.

Alternatively, we could interpret the red colours of the LMC variables as evidence in favour of their identification as Miras. The discrepancy from CLAYTON and FEAST's relation in Fig. 3 may then arise from an incorrect result for their short period group.

To resolve the classification on these variables, as SRd or Mira, spectra may be required, but these would be difficult to obtain for such faint stars.

The author wishes to express his gratitude to Dr. T. LLOYD-EVANS for helpful discussions and to Professor P. A. WAYMAN for his encouragement. He would also like to thank the Dublin Institute for Advanced Studies, and the National Science Council of Ireland for supporting this work.

$$
\text { References: }
$$

BUTLER, C. J., 1972 (a), Dunsink Obs. Publ. In Press.

BUTLER, C. J., 1972 (b) Ir. Astr. J. In press.

CLAYTON, M. L., andFEAST, M. W., 1969, M.N.R.A.S., 146, 411.

FEAST, M. W., 1965, Observatory 85, 16.

HODGE, P. W., and WRIGHT, F. W., 1969, Ap. J., Supp. 17, 467.

KUKARKIN, B. V., KHOLOPÖ, P. N.. EFREMÖV, Yu. N. KUKARKINA, N. P., KUROCHKIN, N. E., MEDVEDEVA, G. I., PEROVA, N. B., FEDOROVICH, V. P., FROLOV, M. S., 1969, General Catalogue of Variable Stars, $3^{\text {rd }}$ edit. Moscow.

LAFLER. I. and KINMAN. T. D., 1965, Ap. J. Supp. XI, 216

I,LOYD-EVANS, T., 1971 , Observatory $91,118$.

PAYNE-GAPOSCHKIN, C., and GAPOSCHKIN, S., 1966, Smithsonian Contr. to Astrophysics, $9,1$.

STOTHERS, R., 1963, A. J. 68, 242.

\section{Discussion to the paper of BUTLER}

ARDEBERG: 1 . Could you say something about the standardization of your magnitudes, and can you estimate how much of a scatter this can introduce?

2. So your standard stars are well distributed both in magnitude and over the plate fields?

BUTLER: 1 . The magnitudes are derived from photometry of $\mathrm{ADH}$ plates calibrated with a large number (approximately 150) of photoelectric standards.

2. The stars cover an area of about two degrees in diameter.

LLOYD EVANS: The bright large amplitude red variables of the SMC show colours (V-I) suggesting type $M$, later at minimum. Spectra of two of them show $\mathrm{H}$ emission lines, with RV of SMC. One (HV 11295) had spectral type M3: at maximum.

SMAK: Is anything known about the spectral types and, in particular, the TiO intensities, of those long-period, relatively bright variables?

BUTLER: They are rather faint for spectroscopic work and so far no spectra have been taken of LMC stars. 